\title{
Position Data Acquisition from NMEA Protocol of Global Positioning System
}

\author{
Hnin Si and Zaw Min Aung
}

\begin{abstract}
Position data acquisition system is described in this paper. The system is based on Magellan Sport Trak GPS, PIC 16F628A microcontroller and personal computer (PC). Position data (latitude and longitude) is extracted from the RMC sentence of the NMEA protocol of the GPS receiver. The extracted data is displayed on personal computer using HyperTerminal communication. The real time received characters can be saved in computer using capture text command in HyperTerminal and retrieved later.
\end{abstract}

Index Terms-data acquisition system; HyperTerminal; NMEA protocol; GPS; latitude; longitude.

\section{INTRODUCTION}

Global Positioning System (GPS) technology has been widely used in a variety of positioning and navigation applications; such as navigation systems for blind people, cellular coverage area determination, autonomous vehicle guidance systems and so on.

\section{A. GPS Receiver}

GPS is a satellite-based global navigation system that enables users to accurately determine 3-dimensional positions (x, y, z) worldwide [1] [6] [7].

GPS consists of 3 segments. They are:

a). The space segment consists of at least 24 satellites. The satellites circle the Earth once every 12 hours. The satellites transmit radio signals continuously to broadcast its changing position and time.

b). The control segment consists of ground stations that monitor and control the satellites.

c). The user segment consists of the user and the GPS receiver. The GPS receiver measures the signals from the satellites and identifies the user's position. The satellites are spaced so that from any point on Earth, at least four satellites will be above the horizon.

Each satellite continually transmits radio signals and broadcasts its position and time. GPS uses satellites in space as reference points to locate the positions on the Earth. The GPS receiver measures the distance from the satellites by measuring the travel time of the radio signals. The distance from the satellite to the GPS receiver is equal to the travel time from the satellite to GPS receiver multiplied by the speed of light. That is, Distance $=$ Travel time $\mathrm{x}$ Speed of light. On the ground, any GPS receiver that contains a computer can locate its own position on the Earth by measuring accurately the distance from three satellites. The

Manuscript received November 10th, 2010.

Hnin Si is with the Department of Materials Science and Materials Engineering Research, MSMERD, Ministry of Science and Technology, Kyaukse, Myanmar (hninsi.81@gmail.com).

Zaw Min Aung is with the Department of Technical and Vocational Education, Ministry of Science and Technology, Nay Pyi Taw, Myanmar (zawminaung@gmail.com). result is provided in the form of a geographic position longitude and latitude.

\section{B. NMEA Sentences}

GPS receiver communication is defined within National Marine Electronics Association (NMEA) specifications. Most computer programs that provide real time position information understand and expect data to be in NMEA format. This data includes the complete PVT (position, velocity, time) solution computed by the GPS receiver. In this research work, the latitude and longitude extraction from NMEA sentences is presented for position determination and navigational purposes.

There are standard NMEA sentences for each device category and there is also the ability to define proprietary sentences for use by the individual company. All of the standard sentences have a two letter prefix that defines the device that uses NMEA sentence type. For GPS receivers the prefix is "GP" which is followed by a three letter sequence that defines the sentence contents [2].

Each NMEA sentence begins with a '\$' and ends with a carriage return or line feed sequence. The data is contained within this single line with data items separated by commas. The data itself is just ASCII text and may extend over multiple sentences in certain specialized instances but is normally fully contained in one variable length sentence. The data may vary in the amount of precision contained in the message.

There are many sentences in the NMEA standard for all kinds of devices that may be used in a Marine environment. Some of the ones that have applicability to GPS receivers are listed below: (all message start with GP.)

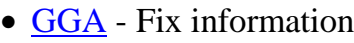

- GLL - Lat/Lon data

- $\underline{\mathrm{GSA}}$ - Overall Satellite data

- GST - GPS Pseudo range Noise Statistics

- GSV - Detailed Satellite data

- $\underline{\mathrm{RMB}}$ - recommended navigation data for GPS

- $\mathrm{RMC}$ - recommended minimum data for GPS

\section{DECODE OF SELECTED NMEA SENTENCES}

The most important NMEA sentences include the GGA which provides the current Fix data, the RMC which provides the minimum GPS sentences information, and the GSA which provides the Satellite status data [2].

$G G A$ - essential fix data which provide 3D location and accuracy data.

\$GPGGA, 123519,4807.038, N, $01131.000, \mathrm{E}, 1,08,0.9,545.4, \mathrm{M}, 46.9$ , $\mathrm{M}$, , * 47

Where: 


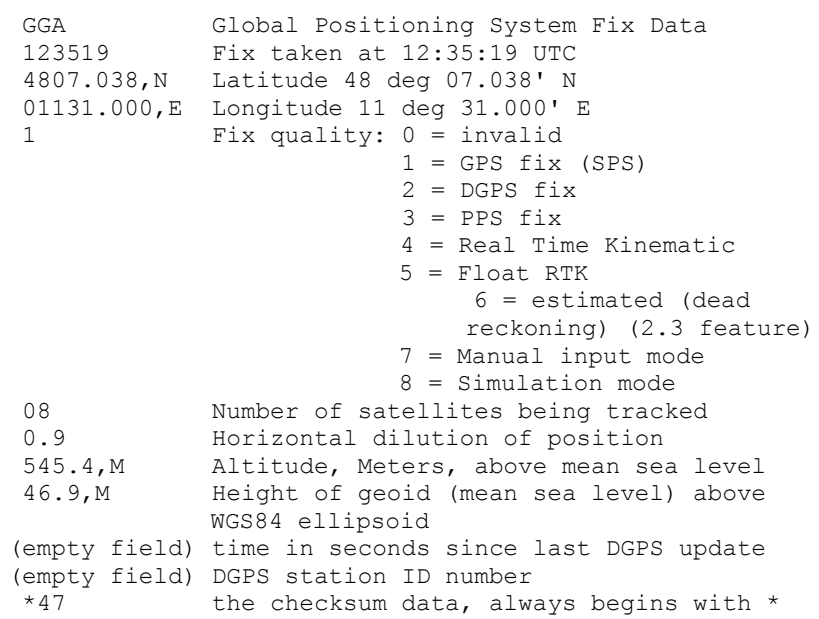

This sentence provides details on the nature of the fix. It includes the numbers of the satellites being used in the current solution and the DOP. DOP (dilution of precision) is an indication of the effect of satellite geometry on the accuracy of the fix.

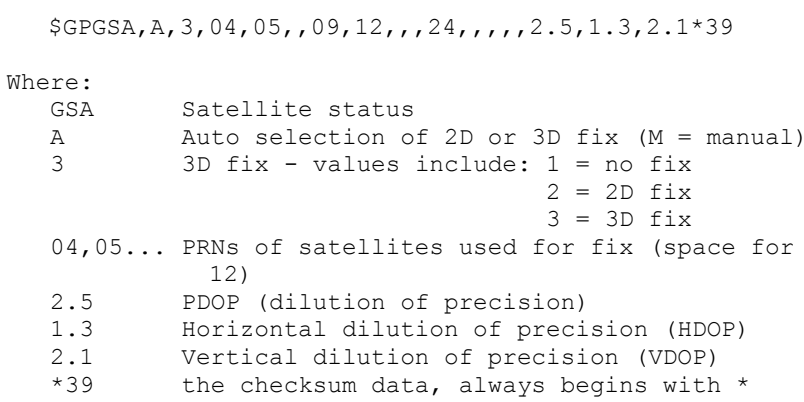

$G S V$ - Satellites in View

This sentence shows data about the satellites that the unit might be able to find based on its viewing mask and almanac data. It also shows current ability to track this data. One GSV sentence only can provide data for up to 4 satellites and thus there may need to be 3 sentences for the full information.

\$GPGSV $, 2,1,08,01,40,083,46,02,17,308,41,12,07,344,39,14,22$ $, 228,45 * 75$

where:

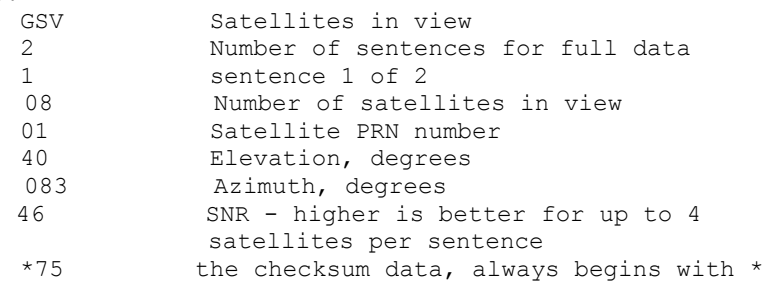

$R M C$ - NMEA has its own version of essential GPS position, velocity and time (PVT) data. It is called RMC, The recommended minimum sentence $\mathrm{C}$.

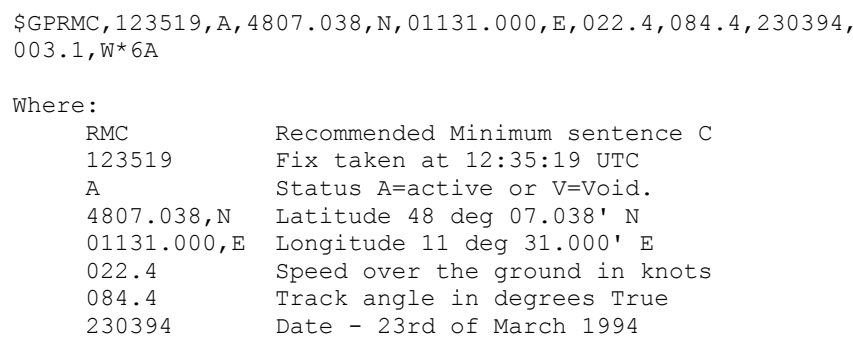

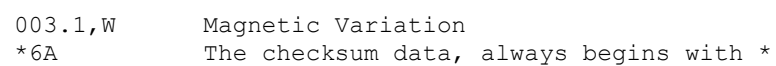

GLL - Geographic Latitude and Longitude

\$GPGLL, 4916.45, N, 12311.12,W, 225444, A, *1D

Where:

GLI

Longitude

4916.46, N Latitude 49 deg. $16.45 \mathrm{~min}$. North

12311.12, w Longitude $123 \mathrm{deg} .11 .12 \mathrm{~min}$. West

225444 Fix taken at 22:54:44 UTC

*iD

Data Active or $\mathrm{V}$ (void)

checksum data

In this research work, Magellan Sport Trak GPS receiver [3] is used.

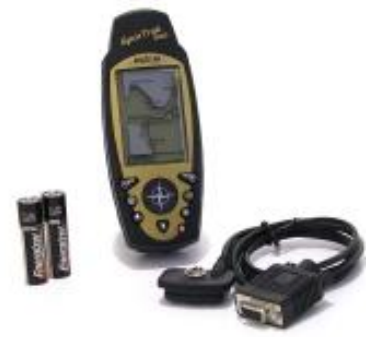

Figure 1. Magellan Sport Trak GPS

\section{OVERVIEW OF THE SYSTEM}

The objective of this research work is to develop the position data acquisition system for navigational purposes. Fig 2 shows the overall system block diagram. The information signals from GPS receiver is transmitted to PIC microcontroller via Maxstream Modems in NMEA format. The microcontroller extracts the position data from the received information signals and sends these position data to personal computer (PC) via RS 232 serial communication. The real time position data (latitude and longitude) is displayed on PC using HyperTerminal. The real time data can be stored in text format and the stored information can be got back later.

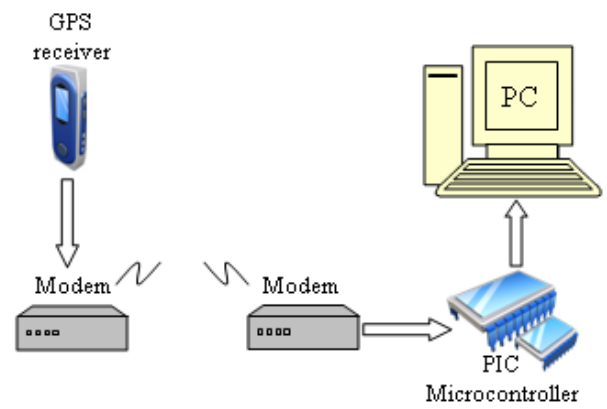

Figure 2. Overall System Block Diagram of the Position Data Acquisition System

\section{SOFTWARE IMPLEMENTATION}

The control algorithm for position acquisition system is set up in the PIC 16F628A microcontroller. The assembly language is used in this project and the MPLAB IDE version 8.10 is used to develop and compile the program. HyperTerminal is used to display the position (latitude and longitude) via RS232 communication with GPS and microcontroller.

Fig 3 shows the microcontroller function algorithm [1] used in this research work. When the program starts, the 
microcontroller initializes the input, output ports and sets baud rates for serial communication. Then it reads ASCII texts from GPS receiver. It waits until GPRMC sentence is received from GPS receiver. Programs that read the data should only use the commas to determine the field boundaries and not depend on column positions. The following subroutine shows how the microcontroller selects the RMC sentence from the information signals of the GPS receiver.

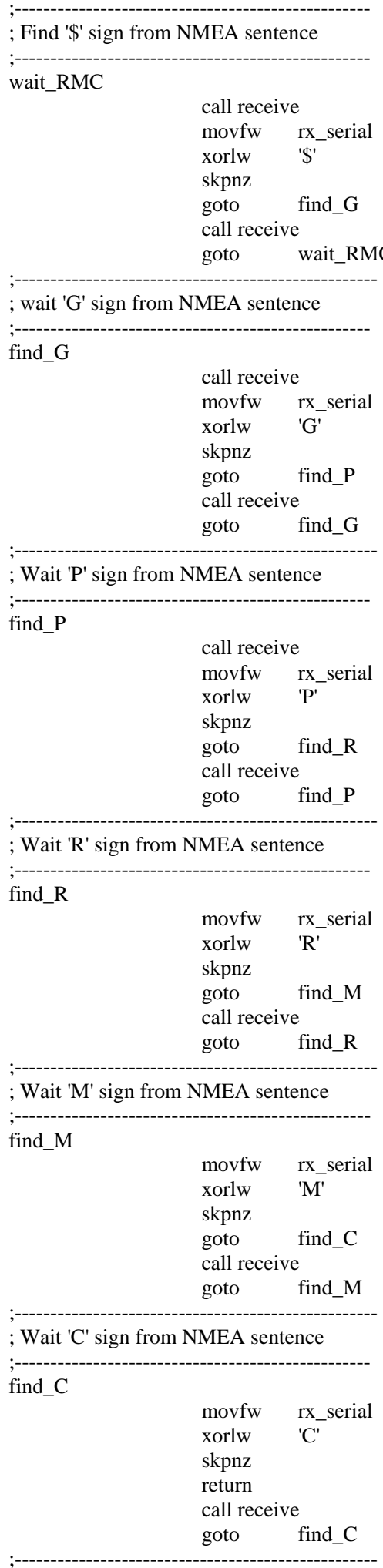

; Wait for comma from NMEA sentence

$\begin{array}{ll}\text { findcomma } & \\ & \text { movfw rx_serial } \\ & \text { xorlw ',' } \\ & \text { skpnz } \\ & \text { return } \\ & \text { call receive } \\ & \text { goto findcomma }\end{array}$

The first three commas of the RMC sentence, which contains the field of UTC time, are skipped in the algorithm. Then the microcontroller reads the next 24 characters of the RMC sentence, which represent latitude and longitude components of the position. By this way, the position data (latitude and longitude) is extracted from RMC sentence. Microcontroller then transmits position data to the personal computer via serial communication. Position data are displayed on PC using HyperTerminal. The same program flow is done to get and display the next real time position data so it is endless loop until the power is off.

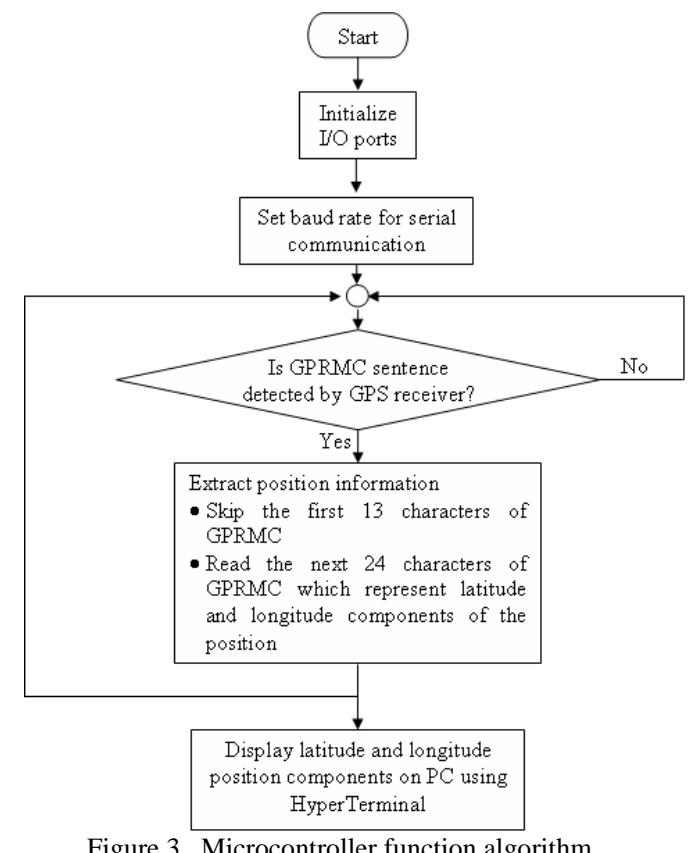

Figure 3. Microcontroller function algorithm

\section{HARDWARE IMPLEMENTATION}

The hardware interface for GPS units is designed to meet the NMEA requirements. They are also compatible with most computer serial ports using RS232 protocols. The interface speed can be adjusted on some models. However, the GPS is configured to transmit at 9600 bit per second in this project. Most GPS receivers will work with Serial to USB adapters and serial ports attached via the pc card adapter. In this research work, DB-9 female and male connectors are used. The $20 \mathrm{MHz}$ crystal oscillator is used to oscillate PIC 16F628A microcontroller. Max-232 IC [4] is used to interface between GPS, microcontroller and personal computer (PC). The DB-9 male connector is used for data acquisition from GPS and DB-9 female connector is used for RS 232 communication to display the position data on PC. The circuit diagram for the data acquisition system is shown in Fig 4. 


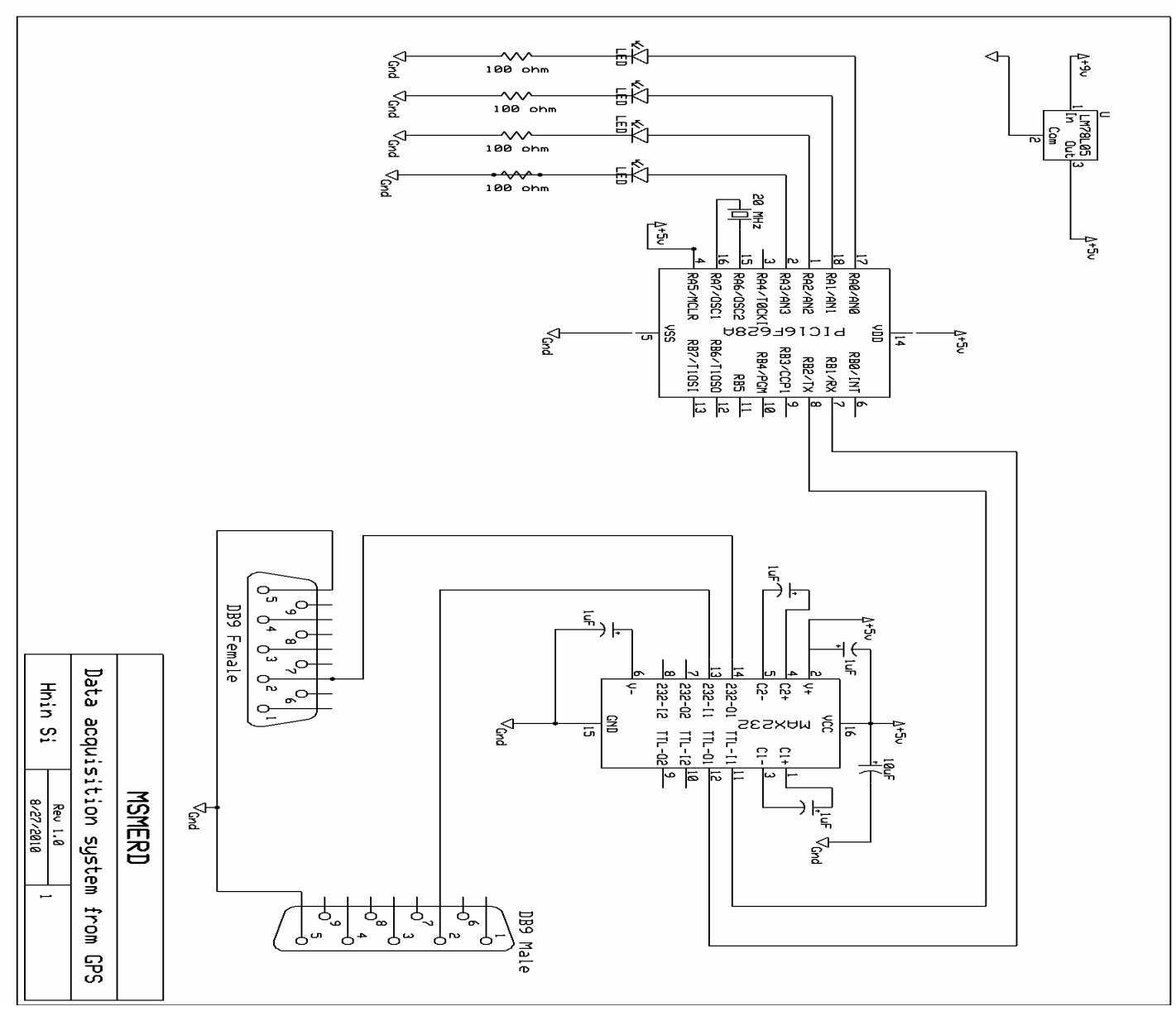

Figure 4. Circuit diagram for the data acquisition system

In the circuit schematics of the system four light emitting diodes (LED) are used. LED connected to RA0 pin is used to check the whether the program is work or not. The next LED connected to RA1 pin will turn on when the serial communication between PC and microcontroller is settled. Another LED connected to RA2 pin will turn on when the GPRMC data from the GPS is received by the microcontroller. The last LED connected to RA3 pin will turn on after the position data has been transmitted to personal computer from microcontroller. The LEDs connected to RA2 and RA3 will turn off after one transmission process has been done and will turn on again for next data acquisition process. These two LEDs will turn on and turn off sequentially. Fig 5 describes the assembled data acquisition system.

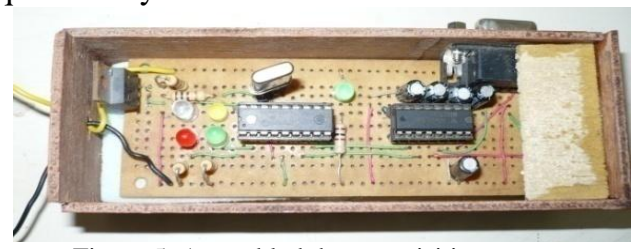

Figure 5. Assembled data acquisition system

\section{TESTS AND RESULTS}

\section{A. Indoor Test}

First of all, the direct serial communication between the GPS and computer has been set up using HyperTerminal with various baud rates. After testing with various baud rates, 9600 bit per second is chosen as this rate is the most suitable rate to acquire the data from the GPS receiver for this research. During indoor test, only PMGNST and GSV sentences are received. PMGNST sentence tells the device's (GPS) status and GSV sentence shows the information about the satellites and there is no RMC sentence. This means that no signal is received related to position inside the building. The received NMEA sentence during indoor test is shown in Fig 6.

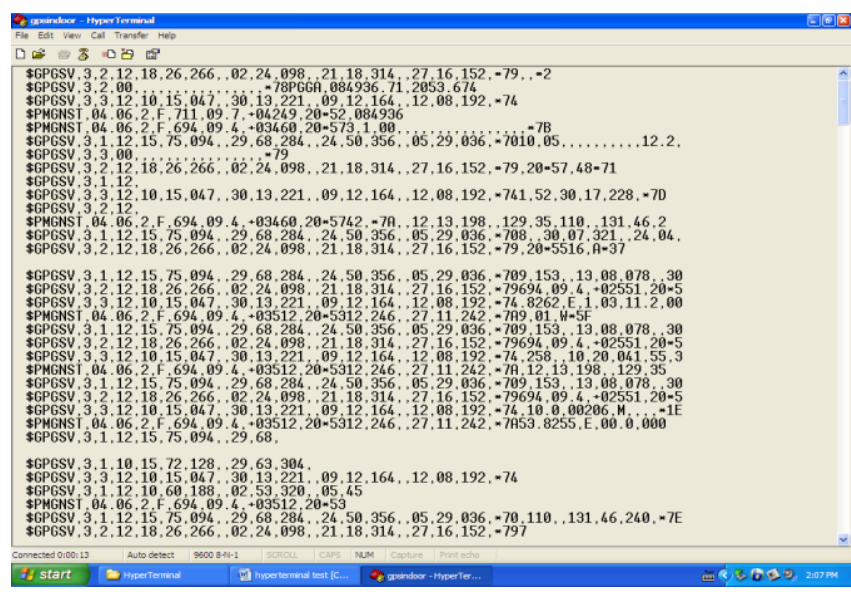

Figure 6. NMEA sentence from GPS during indoor test

\section{B. Outdoor Test}

Next, the outdoor GPS data acquisition is tested using Maxstream Modems. They are used for GPS telemetry. The RS232 data from GPS receiver is input from one end of modem and other end receives the data and connected to the personal computer. The received data displayed on personal 
computer can be seen in Fig 7. In this time, GLL, GGA, GPRMC and GSA sentences are obtained as well as GSV. It means the lat/lon data, fix information, PVT data and overall satellite data are achieved at open-air.

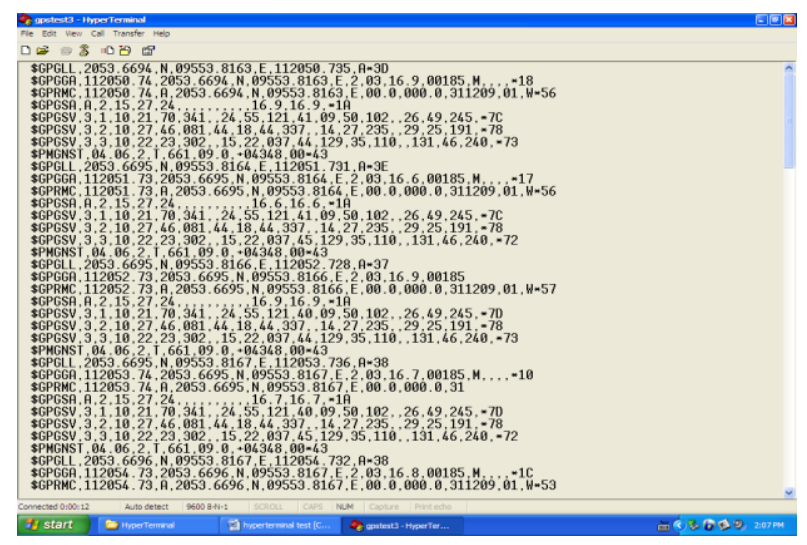

Figure 7. NMEA sentence from GPS during outdoor test

\section{Serial Communication between microcontroller and PC}

Then, the serial communication between PIC 16F628A microcontroller and personal computer is tested. In this case, USART facility of PIC16F628A [5] is used to display data on personal computer. Asynchronous data transmission mode is used. The value of SPBRG register in microcontroller is set as 19 to transmit the data in 9600 baud rate with $20 \mathrm{MHz}$ crystal oscillator. The characters which we want to display are put into the TXREG register of the microcontroller one by one to transmit the characters to PC via RS 232 link. Fig. 8 shows that communication test between microcontroller and personal computer has been successfully set up.

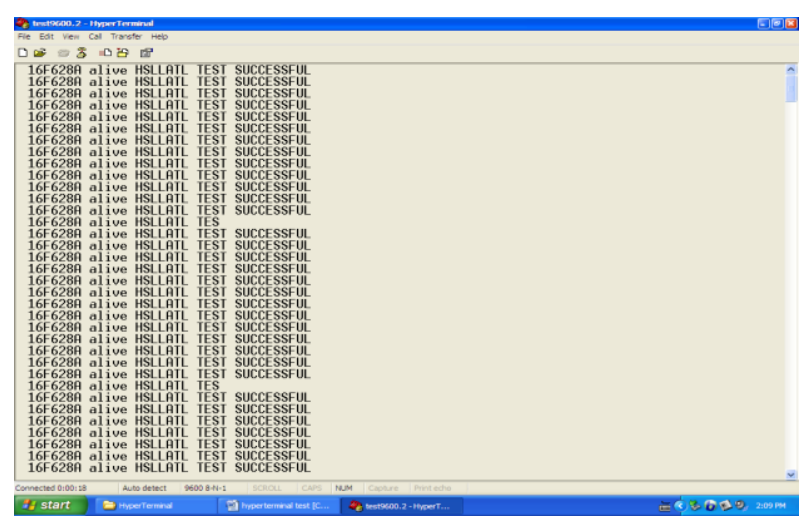

Figure 8. Microcontroller and computer serial communication test

\section{Position data extraction test}

Then, the position data extraction algorithm is developed using MPLAB IDE. The program is tested and modified until the satisfied results are received, then it is burnt into the microcontroller using EPICWIN compiler and EPIC programmer. In this time, RS232 data from GPS receiver is input from one end of modem and other end receives the data and connected to the microcontroller. Microcontroller receives the NMEA sentences and extracts the position data and transmits to the personal computer to display on personal computer. The received extracted data is displayed on the computer as shown in Fig 9.

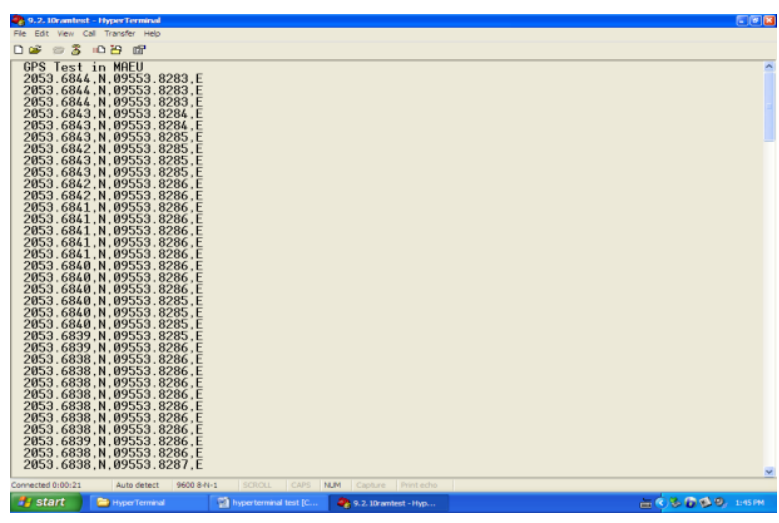

Figure 9. Display of extracted datafrom the GPRMC sentence

\section{E. Real time data storing on PC using HyperTerminal}

As HyperTerminal window can only maintain 500 lines of characters and overwritten when more than 500 lines of characters are received. So the real time received data can be stored using capture text command in the HyperTerminal as shown in Fig 10. The received data can be stored as note pad format in the personal computer and can be retrieved later.

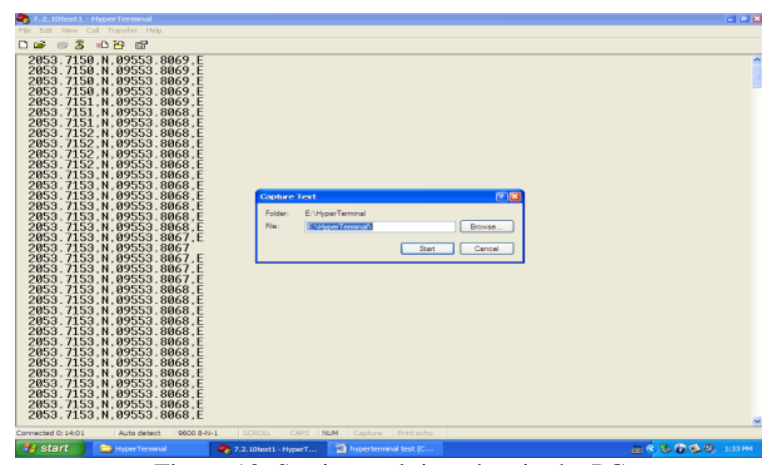

Figure 10. Storing real time data in the PC

\section{CONCLUSION}

This paper discussed about the NMEA sentences of the Global Positioning System and low cost data acquisition system for GPS. According to the results, the position data acquisition system from GPS has been successfully implemented. In the real case of navigation systems, the heading data is required as well as latitude and longitude values to analyze the trajectory plan for navigation. The heading value can be got by reading more characters from GPRMC sentence since it contains heading information also.

\section{REFERENCES}

[1] AmeerAmeer H. Morad, "GPS Talking for Blind People", Journal of Emerging Technologies in Web Intelligence, Volume 2, No.3, August 2010.

[2] http://aprs.grids.nl./nmea/

[3] SporTrak User Manual, Magellan. (C) 2002 by Thales Navigation

[4] Max 232, Max 232I Dual EIA 232 drivers and receiver, data sheet, SLLS0471 - February 1989 - revised October 2002, Texas Instruments.

[5] PIC 16F627A/628A/648A data sheet, Microchip, (c) 2005 Microchip Technology Inc.

[6] Elliott D Kaplan and Christopher Hegarty,"Understanding GPS: Principles and Applications", Artech House,2nd ed., 2006.

[7] James Bao-Yen Tsui, "Fundamentals of Global Positioning System Receivers: A Software Approach", 2nd ed., Wiley, 2005.

[8] S. Omar, A. Liu, P. Techateerawat, "Navigation Wireless LAN Device", The 2004 International Symposium on GNSS/GPS, Sydney, Australia, 6-8 December 2004 